\title{
TENSILE PROPERTIES OF SOME STRUCTURAL ALLOY STEELS AT HIGH TEMPERATURES
}

\author{
By H. J. French
}

\section{ABSTRACT}

The report gives results of determination of tensile strength, proportional limit, elongation, reduction of area, and strength at fracture throughout the range 20 to $55^{\circ} \mathrm{C}$ for four steels containing about $0.3^{8}$ per cent carbon, as follows: (a) Plain carbon steel; (b) $3 \frac{1}{2}$ per cent nickel steel; (c) 3 per cent nickel, I per cent chromium steel; (d) I per cent chromium, 0.20 per cent vanadium steel.

Brief reference is made to the type of fractures obtained in testing steels at various temperatures, and particular attention is paid to comparison of the tensile properties of these alloys at $55^{\circ} \mathrm{C}$.

\section{CONTENTS}

Page

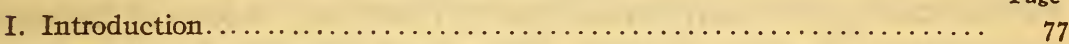

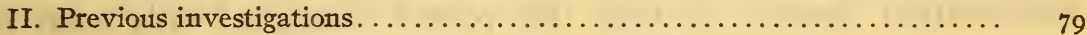

III. Materials and methods used $\ldots \ldots \ldots \ldots \ldots \ldots \ldots \ldots \ldots \ldots \ldots \ldots \ldots \ldots \ldots \ldots \ldots \ldots$

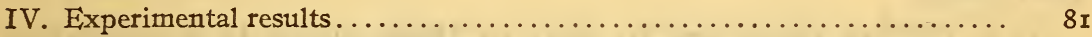

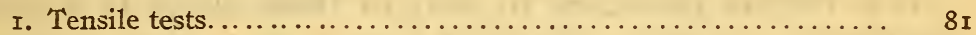

(a) Tensile strength............................. 8 I

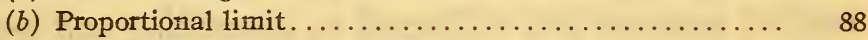

(c) Elongation and reduction of area................ 89

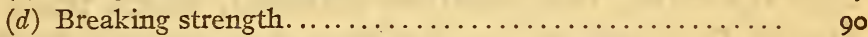

2. Microscopic examination of fractures................... go

V. Summary............................

\section{INTRODUCTION}

In the attempted production of ammonia in the United States by the Haber process ${ }^{1}$ gradual elongation and ultimate fracture of bolts in converter chamber heads have introduced serious operating difficulties. Under a mean temperature of $550^{\circ} \mathrm{C}$ to which heads, nuts, and bolts were subjected the elongation of the latter was such as to require tightening of the nuts almost daily. The use of some of the more commonly employed structural alloy steels did not remove this difficulty, and a search of the literature revealed little exact information on which a comparison of the high temperature tensile properties of various alloy steels could be based.

In view of the fact that an investigation of the resistance to corrosion by ammonia of various ferrous and nonferrous alloys

1 R.S. Tour, "The direct synthetic ammonia process," Journ. Ind. and Eng. Chem., 12, No. 9, p. 844; September, 1920. 
was in progress in connection with redesign of operating equipment by the Ordnance Department, the Bureau of Standards was requested to undertake determination of the tensile properties of a number of structural alloy steels throughout the temperature range 20 to $550^{\circ} \mathrm{C}\left(70\right.$ to $\left.1020^{\circ} \mathrm{F}\right)$. From the combined results of investigation of resistance to corrosion of various metals and the tensile properties of steels at high temperatures it was hoped that selection of a suitable alloy for withstanding required stresses could be made, and if this proved unsatisfactory as regards resistance to attack by the gases encountered at least a more suitable metal lining for the converters could be installed.

The original plan of procedure included tests of a plain carbon steel and a number of structural alloy steels, most of which were of acknowledged industrial importance. However, because of the desire to keep variations in carbon content at a minimum, diffculty was encountered in obtaining some of the alloys, notably carbon-manganese and chromium-molybdenum steels, of the exact composition desired, so that this report comprises only tests of four steels.

It is further restricted to tests of these alloys in a normalized condition, as data so obtained form a basis of comparison with tests of samples first subjected to quenching and tempering. The high operating temperatures attained, which reach about $650^{\circ} \mathrm{C}$ $\left(\mathrm{r} 200^{\circ} \mathrm{F}\right)$, limit the tempering subsequent to hardening to relatively high temperatures, while the large dimensions of the converter heads and some of the other parts of the equipment subjected to high temperatures, which would introduce difficulties in quenching, make the use of a forged or normalized steel highly desirable.

Shortly after completion of the tests about to be described tensile properties at high temperatures of a number of carbon and alloy steels subjected to varying thermal treatments were reported by MacPherran, ${ }^{2}$ and in discussion of this paper by Spooner considerable additional data were presented. However, the work of MacPherran and Spooner was confined, in so far as the elastic properties are concerned, to determination of yield point; whereas the tests carried out by the present author include determinations of the limit of proportionality and strength at fracture, and the interpretation of these data is somewhat different from that given by either of the authors mentioned.

${ }^{2}$ R. S. MacPherran, "Comparative tests of steels at high temperatures," Proc. Amer. Soc. for Testing Materials; $192 x$. 


\section{PREVIOUS INVESTIGATIONS}

As already indicated, there was until quite recently a scarcity of accurate data on the high temperature tensile properties of various structural alloy steels. This is still the case as regards the effect of temperature on the limit of proportionality, of importance in design of engineering equipment, and in addition there is some disagreement between results reported by different investigators for similar alloys. Bregowsky and Spring ${ }^{3}$ determined yield point, tensile strength, elongation, and reduction of area from 20 to $550^{\circ} \mathrm{C}$ (70 to $1020^{\circ} \mathrm{F}$ ) for rolled 30 per cent nickel steel.

In I9I3 Schulz ${ }^{4}$ reviewed available data relating to the high temperature properties of turbine materials, but the information presented for alloy steels was, in large part, for those containing varying proportions of nickel.

Guillet $^{5}$ reported, among several special alloys for use at high temperatures, a steel containing nickel, chromium, and tungsten which showed exceptionally high strength at 750 to $800^{\circ} \mathrm{C}$ ( 1380 to $\left.1470^{\circ} \mathrm{F}\right)$.

A comprehensive analysis of the effects of high temperatures on hardness, tensile and impact properties, freedom from warpage, etc., of steels containing 3 per cent nickel and various proportions of chromium, high-speed steels and various chromium and chromium-cobalt steels was recently prepared by Gabriel. ${ }^{\circ}$ As regards high tensile strength at elevated temperatures, these steels were considered of value in the following order: (I) High tungsten; (2) high chromium, high carbon; (3) high chromium, low carbon; (4) 3 per cent nickel and nickel-chromium.

In the selection of steels for valves the author recommended the different type alloys, as follows: (I) A tungsten steel, (2) a steel high in chromium, (3) a nickel steel.

$\mathrm{MacPherran}^{7}$ recently reported tensile tests from 20 to about $650^{\circ} \mathrm{C}\left(70\right.$ to $\mathrm{I} 200^{\circ} \mathrm{F}$ ) for a variety of alloy steels under different heat treatments, as shown in Table $\mathrm{I}$. In discussion of this paper A. P. Spooner presented a large number of tests at temperatures up to about $870^{\circ} \mathrm{C}\left(1600^{\circ} \mathrm{F}\right)$ for the different steels listed in

3 I. M. Bregowsky and I. W. Spring, "The effect of high temperature on the physical properties of some alloys," Proc. Int. Assoc. for Testing Materials, VI Congress, VII 1 ; Igr2.

"Schulz, "Neuere versuche und erfahrungen mit turbinenschaufelmaterial für höhe temperaturen," Die Turbine, 9, p. 243; 1913.

${ }^{5}$ L. Guillet, "Alloys having remarkable properties at very high or very low temperatures," Rev. Mfet. 11, p. $969 ;$ igI 4 .

6 G. Gabriel, "Comparative values of motor valve steels," Iron Age, 106, p. I465; I920.

${ }^{7}$ See note 2. 
Table 2 and, in general, confirmed the work of MacPherran where direct comparisons were possible.

TABLE 1.-Alloy Steels Tested at High Temperatures $a$

\begin{tabular}{|c|c|c|c|c|c|}
\hline \multicolumn{5}{|c|}{ Type coraposition (per cent) } & \multirow{2}{*}{ Condition in which steel was tested } \\
\hline C & $\mathrm{Mn}$ & $\mathrm{Ni}$ & $\mathrm{Cr}$ & $\mathrm{V}$ & \\
\hline 0.21 & & 3. 25 & & & Annealed at $802^{\circ} \mathrm{C}\left(1475^{\circ} \mathrm{F}\right)$ \\
\hline .25 & & 33.9 & & & Do. \\
\hline .34 & & 2. 38 & 0.38 & & $816^{\circ} \mathrm{C}\left(1500^{\circ} \mathrm{F}\right)$ water, $705^{\circ} \mathrm{C}\left(1300^{\circ} \mathrm{F}\right)$ \\
\hline .53 & & 20. 78 & 7.42 & & As rolled \\
\hline .30 & & & .83 & 0.17 & $857^{\circ} \mathrm{C}\left(1575^{\circ} \mathrm{F}\right)$ water, $732^{\circ} \mathrm{C}\left(1350^{\circ} \mathrm{F}\right)$ \\
\hline .69 & & & 3.36 & & $843^{\circ} \mathrm{C}\left(1550^{\circ} \mathrm{F}\right)$ water, $705^{\circ} \mathrm{C}\left(1300^{\circ} \mathrm{F}\right)$ \\
\hline .72 & 0.92 & & & & Annealed at $802^{\circ} \mathrm{C}\left(1475^{\circ} \mathrm{s}\right)$ \\
\hline .82 & & & .91 & .18 & $816^{\circ} \mathrm{C}\left(1500^{\circ} \mathrm{F}\right)$ water, $705^{\circ} \mathrm{C}\left(1500^{\circ} \mathrm{F}\right)$ \\
\hline
\end{tabular}

a MacPherran, Amer. Soc. for Testing Materials; x92x.

TABLE 2.-Alloy Steels Tested at High Temperatures $a$

\begin{tabular}{|c|c|c|c|c|c|c|}
\hline \multicolumn{6}{|c|}{ Type composition (per cent) } & Concition in which steel was tested \\
\hline C & $\mathrm{Ni}$ & $\mathrm{Cr}$ & $\mathrm{V}$ & Mo & $\mathrm{W}$ & . \\
\hline 0.10 & .. & 1 & & & & Normalized $870^{\circ} \mathrm{C}\left(1600^{\circ} \mathrm{F}\right)$ \\
\hline .45 & & & & & & Do. \\
\hline .33 & 3.5 & $\ldots \ldots$ & & & & 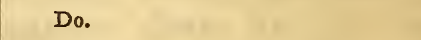 \\
\hline .38 & 2.0 & 1.0 & & & & Do. \\
\hline .37 & .... & 1.0 & 0.20 & $\cdots \cdot$ & & Do. \\
\hline .47 & ... & 1.0 & ...... & 0.35 & & Do. \\
\hline 1.10 & ... & .50 & .20 & & & Annealed $740^{\circ} \mathrm{C}\left(1360^{\circ} \mathrm{F}\right)$ \\
\hline .48 & & 1.3 & .20 & & & Annealed $730^{\circ} \mathrm{C}\left(1350^{\circ} \mathrm{F}\right)$ \\
\hline .25 & $\cdots$ & 14. 0 & ..... & & $\cdots$ & Annealed $790^{\circ} \mathrm{C}\left(1450^{\circ} \mathrm{F}\right)$ \\
\hline .66 & ... & 3.2 & .70 & & 15.9 & Annealed $905^{\circ} \mathrm{C}\left(1660^{\circ} \mathrm{F}\right)$ \\
\hline .20 & & 31.0 & & & & Annealed $760^{\circ} \mathrm{C}\left(1400^{\circ} \mathrm{F}\right)$ \\
\hline
\end{tabular}

a A. P. Spooner, Proc. Amer. Soc. for Testing Materials; r92x.

These data, while the most recently presented, are together the most comprehensive compilation of tests so far recorded for the modern types of structural alloy steels, but consist of determinations of tensile strength, elongation, reduction of area, and, in part of the temperature ranges given, values for yield point. 


\section{MATERIALS AND METHODS USED}

The four steels tested were made by the electric furnace process at the plant of the Halcomb Steel Co., Syracuse, N. Y., and supplied through the Nitrate Division, Ordnance Department, in the form of $\mathrm{I}$ by $\mathrm{I} / 2$ inch hot-rolled bars of the compositions shown in Table 3. After cutting into desired lengths for test specimens the bars were normalized by heating to 800 or $850^{\circ} \mathrm{C}$ (Table 3) and cooled in still air. They were then machined to the form and dimensions shown in Fig. I. All tests were made with the special apparatus devised by the author and in the manner previously described $^{8}$ in detail.

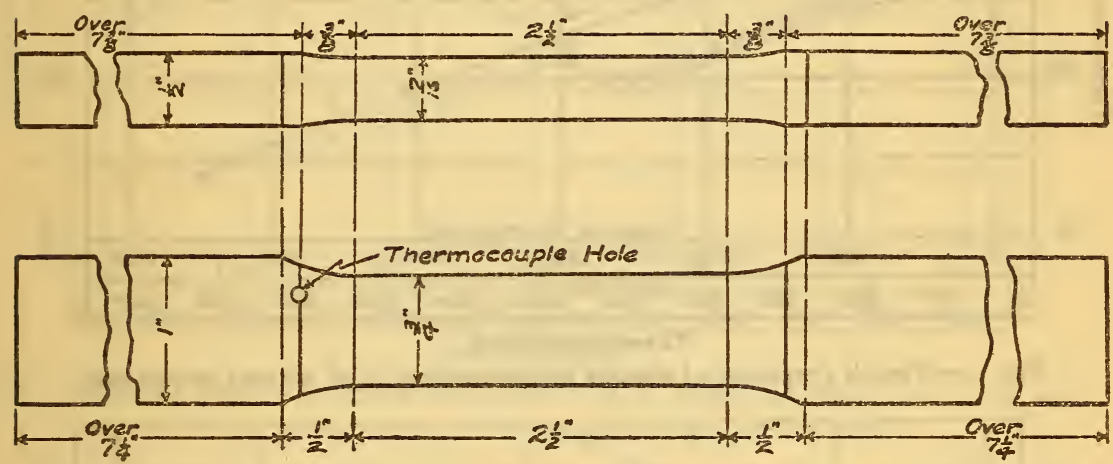

FIG. I.-Form and dimensions of test specimen used

TABLE 3.-Composition and Heat Treatment of Steels Tested

\begin{tabular}{|c|c|c|c|c|c|c|c|c|c|}
\hline \multirow[b]{2}{*}{ Steel } & \multicolumn{8}{|c|}{ Composition (per cent) } & \multirow{2}{*}{$\begin{array}{l}\text { Heat treatment } \\
\text { Heated for } 30 \\
\text { minutes at tem- } \\
\text { erature desig. } \\
\text { nated and cooled } \\
\text { in still air }\end{array}$} \\
\hline & C & $\mathrm{Mn}$ & $\mathbf{P}$ & $\widehat{S}$ & $\mathrm{~S} 1$ & Ni & $\mathrm{Cr}$ & V & \\
\hline A.. & 0.38 & 0.56 & 0.014 & 0.013 & 0.14 & -. & & & $850^{\circ} \mathrm{C}$ \\
\hline B. & .37 & .67 & .021 & .010 & .20 & 3. 43 & $\ldots \ldots$ & 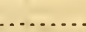 & $800^{\circ} \mathrm{C}$ \\
\hline C. . & .39 & .59 & .019 & .009 & .23 & 3.05 & 0.93 & & $850^{\circ} \mathrm{C}$ \\
\hline D. . & .37 & .74 & .020 & .023 & .21 & $\ldots \ldots \ldots$ & 1.04 & 0.17 & $850^{\circ} \mathrm{C}$ \\
\hline
\end{tabular}

\section{EXPERIMENTAL RESULTS}

\section{TENSILE TESTS}

(a) Tensile STrengTh.-The results of tensile tests at various temperatures throughout the range 20 to $550^{\circ} \mathrm{C}$ (70 to $1020^{\circ} \mathrm{F}$ ) are given in Tables 4, 5, 6, and 7, and represented graphically in Figs. 2 to 5 , inclusive.

${ }^{8}$ Forthcoming Bureau Tech. Paper: Effect of Temperature Deformation and Rate of Loading on the Tensile Properties of Low Carbon Steel Beiow the Thermal Critical Range. Also H. J. French, "Tensile properties of boiler plate at elevated temperatures," Min. and Met., 158, sec. 15; 1920. 


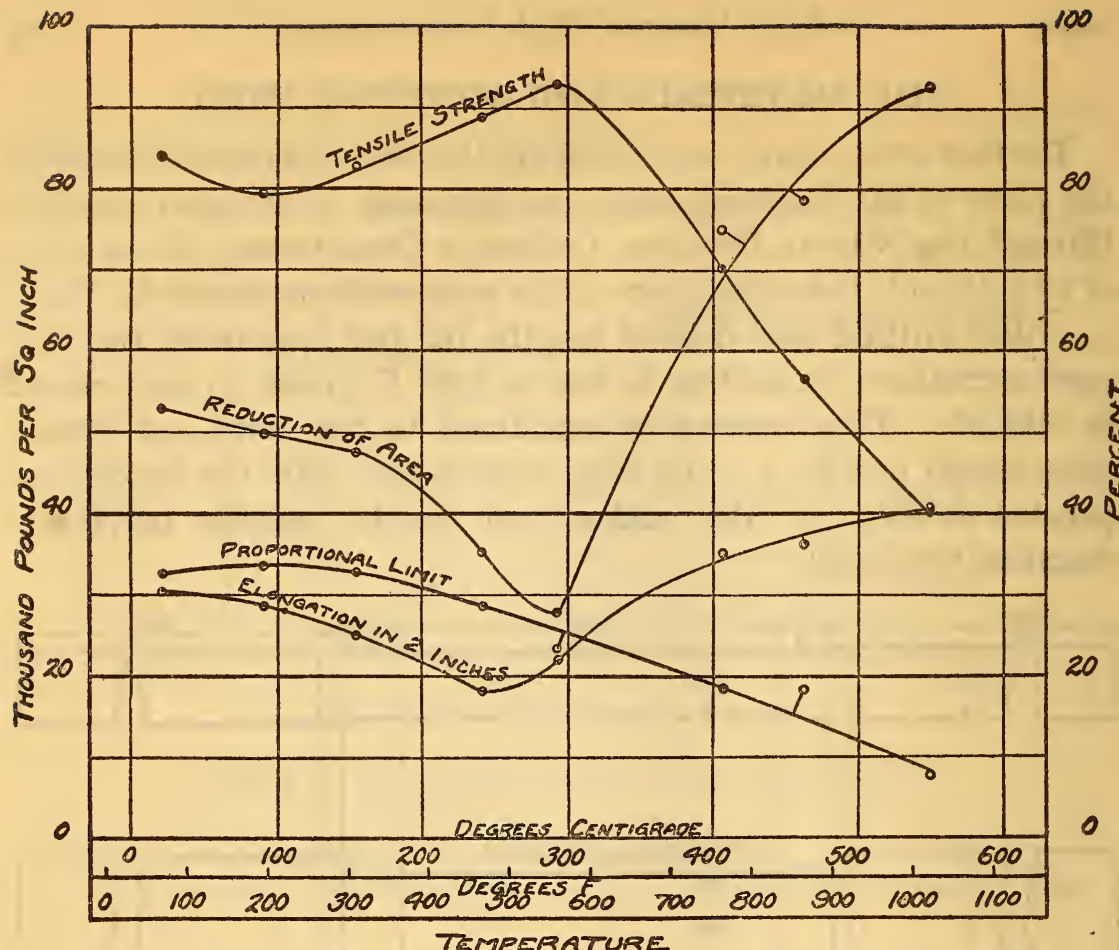

FrG. 2.-Tensile properties at elevated temperatures of 0.38 per cent carbon steel

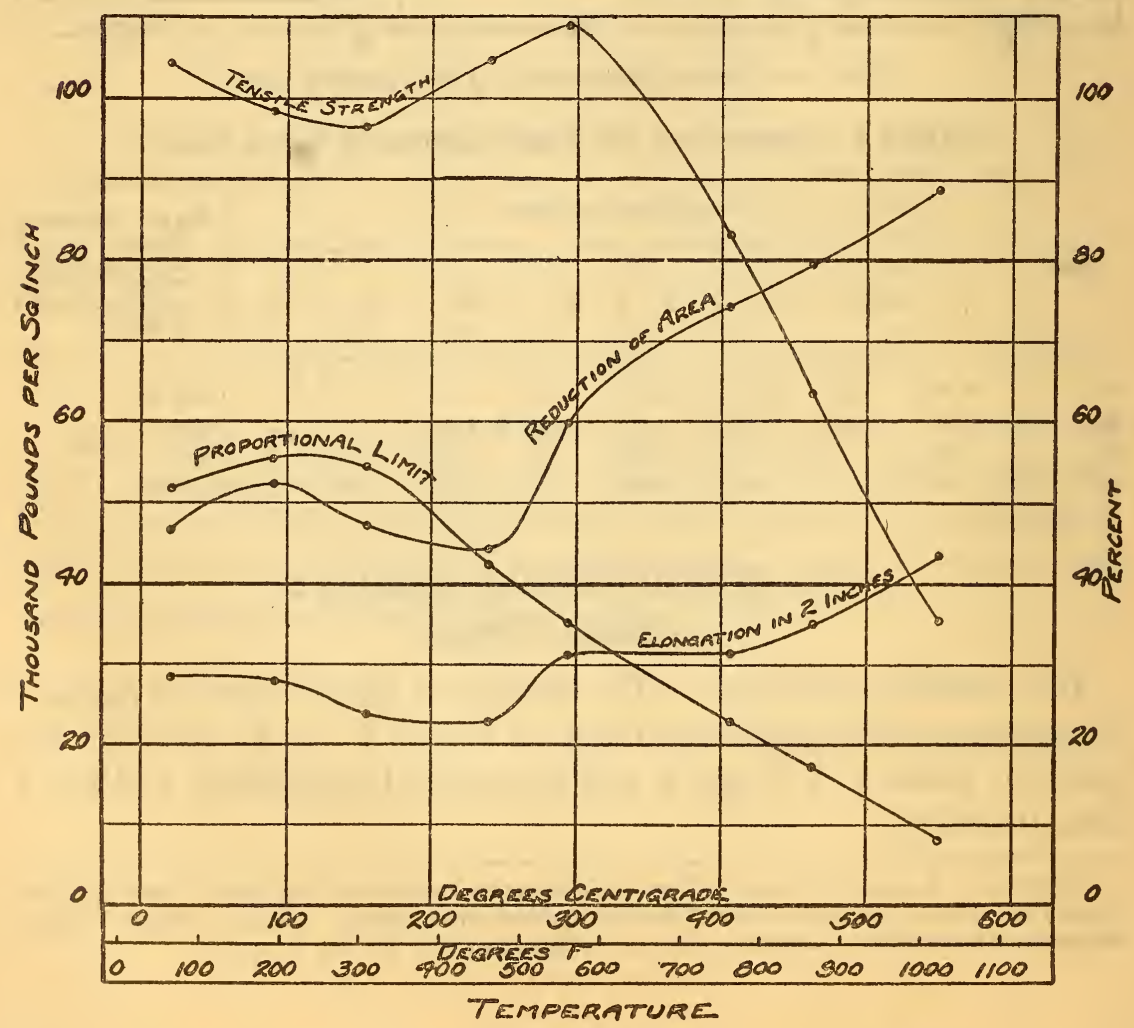

Fig. 3.-Tensile properties at elevated temperatures of $3 \mathrm{~T} / 2$ per cent nickel steel containing 


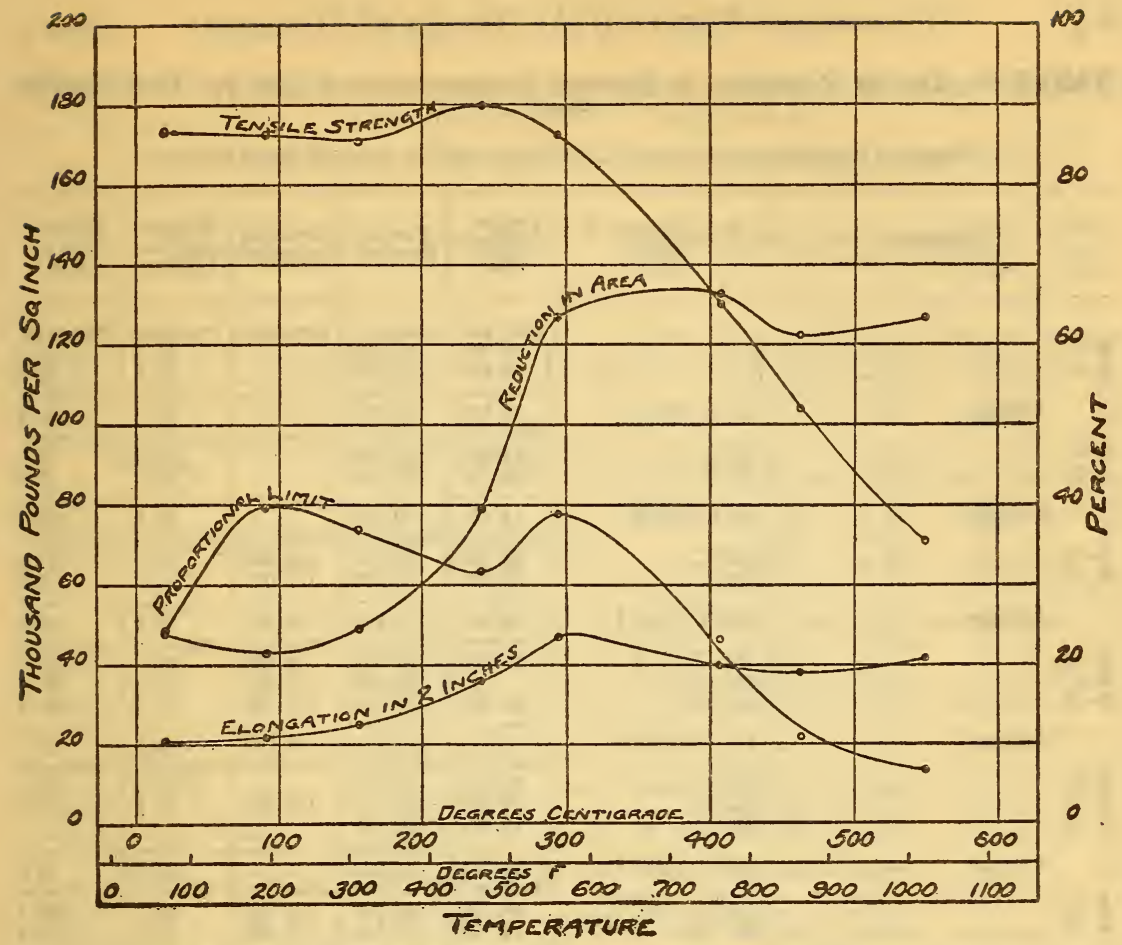

Fig. 4.-Tensile properties at elevated iemperatures of nickel-chromium steel o the type 3 per cent nickel, I per cent chromium, and 0.4 per cent carbon

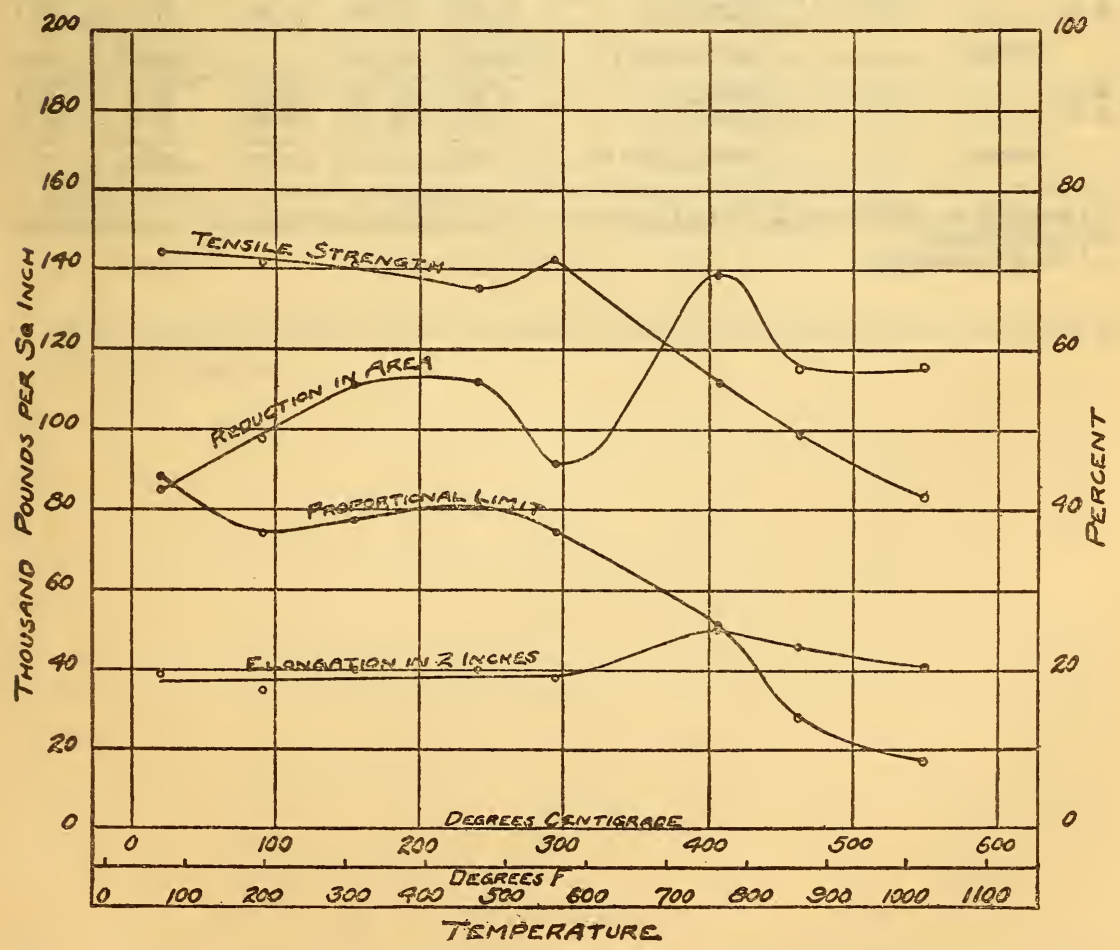

FIG. 5.-Tensile properties at elevated temperatures of chromium-vanadium steel of the type I per cent chromium, 0.2 per cent vanadium, and 0.37 per cent carbon $62954^{\circ}-21-2$ 
TABLE 4.-Tensile Properties at Elevated Temperatures of $0^{\circ} 38$ Per Cent Carbon Steel

[Chemical composition (per cent): C, 0.35; Mn, 0.55; P, 0.014; S, 0.013; Si, 0.14]

\begin{tabular}{|c|c|c|c|c|c|c|}
\hline Number & $\begin{array}{c}\text { Temperature of } \\
\text { test }\end{array}$ & $\begin{array}{l}\text { Propor- } \\
\text { tional } \\
\text { limit }\end{array}$ & $\begin{array}{l}\text { Tensile } \\
\text { strength }\end{array}$ & $\begin{array}{l}\text { Breaking } \\
\text { strengtha }\end{array}$ & $\begin{array}{l}\text { Elonga- } \\
\text { tion in } \\
2 \text { inches }\end{array}$ & $\begin{array}{l}\text { Reduc- } \\
\text { tion of } \\
\text { area }\end{array}$ \\
\hline$A-1 \ldots \ldots \ldots$. & $\left(b^{(}\right)$. & $\begin{array}{l}\text { Lbs./in.2 } \\
\begin{array}{r}34900 \\
30500\end{array}\end{array}$ & $\begin{array}{r}\text { Lbs./in. }{ }^{2} \\
85200 \\
83200 \\
\end{array}$ & \begin{tabular}{|l} 
Lbs./in. ${ }^{2}$ \\
\hdashline$\ldots \ldots$ \\
\end{tabular} & $\begin{array}{r}\text { Per cent } \\
27.5 \\
33.5 \\
\end{array}$ & $\begin{array}{r}\text { Per cent } \\
52.3 \\
53.3 \\
\end{array}$ \\
\hline Average........ & $21^{\circ} \mathrm{C}\left(70^{\circ} \mathrm{F}\right) \ldots$ & 32700 & 84200 & $\cdots$ & 30.5 & 52.8 \\
\hline $\begin{array}{l}\mathbf{A}-6 \ldots \\
\mathbf{A}-9 \ldots\end{array}$ & $\begin{array}{l}92^{\circ} \mathrm{C} \ldots \ldots \ldots \\
92^{\circ} \mathrm{C} \ldots \ldots \ldots . . .\end{array}$ & $\begin{array}{ll}34 & 000 \\
33 & 700\end{array}$ & $\begin{array}{l}79400 \\
79500\end{array}$ & - & $\begin{array}{l}28.0 \\
29.5\end{array}$ & $\begin{array}{l}47.6 \\
52.2\end{array}$ \\
\hline Average.......... & $92^{\circ} \mathrm{C}\left(198^{\circ} \mathrm{F}\right) .$. & 33850 & 79450 & ..... & 28.8 & 49.9 \\
\hline 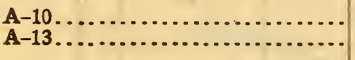 & $\begin{array}{l}155^{\circ} \mathrm{C} \ldots \ldots \ldots \ldots \\
155^{\circ} \mathrm{C} \ldots \ldots \ldots \ldots\end{array}$ & $\begin{array}{ll}33 & 250 \\
32 & 750\end{array}$ & $\begin{array}{ll}85 & 500 \\
80 & 200\end{array}$ & $\begin{array}{l}140000 \\
137000\end{array}$ & $\begin{array}{l}24.5 \\
26.0\end{array}$ & $\begin{array}{l}47.2 \\
48.3\end{array}$ \\
\hline Average. ........ & $155^{\circ} \mathrm{C}\left(311^{\circ} \mathrm{F}\right) \ldots$ & 33000 & 82850 & 138500 & 25.2 & 47.8 \\
\hline $\begin{array}{l}\text { A-12 } 12 \ldots \ldots \ldots \\
\text { A-17 } \ldots \ldots \ldots \\
\mathbf{A}-18 \ldots \ldots \ldots\end{array}$ & $\begin{array}{l}241^{\circ} \mathrm{C} \ldots \ldots \ldots \ldots \\
241^{\circ} \mathrm{C} \ldots \ldots \ldots \ldots \\
241^{\circ} \mathrm{C} \ldots \ldots \ldots \ldots\end{array}$ & $\begin{array}{l}27500 \\
30000\end{array}$ & $\begin{array}{l}87500 \\
90400 \\
88900\end{array}$ & $\begin{array}{l}129000 \\
127400 \\
129700\end{array}$ & $\begin{array}{l}19.0 \\
18.0 \\
17.5\end{array}$ & $\begin{array}{l}37.0 \\
32.7 \\
36.9\end{array}$ \\
\hline Average......... & $241^{\circ} \mathrm{C}\left(466^{\circ} \mathrm{F}\right) \ldots$ & 28750 & 88950 & 128700 & 18.2 & 35.5 \\
\hline $\begin{array}{l}\mathbf{A}-8 \ldots \ldots \\
\mathbf{A}-11 \ldots \ldots \\
\mathbf{A}-19 \ldots \ldots\end{array}$ & $\begin{array}{l}292^{\circ} \mathrm{C} \ldots \ldots \ldots \\
294^{\circ} \mathrm{C} \ldots \ldots \ldots \\
294^{\circ} \mathrm{C} \ldots \ldots \ldots\end{array}$ & $\begin{array}{ll}26 & 000 \\
24 & 500 \\
20 & 000\end{array}$ & $\begin{array}{ll}91 & 500 \\
93 & 500 \\
94 & 000\end{array}$ & $\begin{array}{r}116700 \\
\cdots\end{array}$ & $\begin{array}{l}24.0 \\
20.0 \\
\cdots\end{array}$ & $\begin{array}{r}28.6 \\
27.5 \\
\ldots \ldots\end{array}$ \\
\hline Average.......... & $293^{\circ} \mathrm{C}\left(559^{\circ} \mathrm{F}\right) \ldots$ & 23500 & 93000 & …...... & 22.0 & 28.0 \\
\hline $\begin{array}{l}\mathbf{A}-7 \\
\mathbf{A}-15 \ldots \ldots\end{array}$ & $\begin{array}{l}407^{\circ} \mathrm{C} \ldots \ldots \ldots \ldots \\
407^{\circ} \mathrm{C} \ldots \ldots \ldots \ldots\end{array}$ & $\begin{array}{ll}18 & 500 \\
18 & 500\end{array}$ & $\begin{array}{ll}68 & 000 \\
72 & 500\end{array}$ & $\begin{array}{l}137700 \\
145800\end{array}$ & $\begin{array}{l}36.5 \\
34.0\end{array}$ & $\begin{array}{l}76.2 \\
73.8\end{array}$ \\
\hline Average......... & $407^{\circ} \mathrm{C}\left(765^{\circ} \mathrm{F}\right) \ldots$ & 18500 & 70250 & 141750 & 35.2 & 75.0 \\
\hline $\begin{array}{l}\text { A-3.... } \\
\text { A-16... }\end{array}$ & $\begin{array}{l}463^{\circ} \mathrm{C} \ldots \ldots \ldots \\
463^{\circ} \mathrm{C} \ldots \ldots \ldots\end{array}$ & $\begin{array}{ll}21400 \\
15300\end{array}$ & $\begin{array}{ll}57 & 300 \\
56 & 000 \\
\end{array}$ & 123700 & $\begin{array}{l}35.5 \\
37.0\end{array}$ & $\begin{array}{l}78.1 \\
79.2\end{array}$ \\
\hline Average .................... & $463^{\circ} \mathrm{C}\left(865^{\circ} \mathrm{F}\right) \ldots$ & 18350 & 56650 & $\ldots \ldots$ & 36.2 & 78.6 \\
\hline $\begin{array}{l}\text { A-21. } \\
\text { A-22. }\end{array}$ & $\begin{array}{l}550^{\circ} \mathrm{C} \ldots \ldots . . . \\
550^{\circ} \mathrm{C} \ldots \ldots . .\end{array}$ & $\begin{array}{ll}9500 \\
6000\end{array}$ & $\begin{array}{ll}41 & 700 \\
38 & 150\end{array}$ & $\begin{array}{l}133200 \\
100300\end{array}$ & $\begin{array}{l}38.0 \\
43.0\end{array}$ & $\begin{array}{l}93.0 \\
92.5\end{array}$ \\
\hline Average... & $550^{\circ} \mathrm{C}\left(1022^{\circ} \mathrm{F}\right) \ldots$ & 7750 & 39950 & 116750 & 40.5 & 92.7 \\
\hline
\end{tabular}

a Breaking strength is taken as the load observed at fracture (in pounds) divided by the reduced area (in square inches).

$b$ Room temperature. 
TABLE 5.-Tensile Properties at Elevated Temperatures of $3 \frac{1}{2}$ Per Cent Nickel Steel Containing 0.37 Per Cent Carbon

[Chemical composition (per cent): $\mathrm{C}, 0.37 ; \mathrm{Mn}, 0.67 ; \mathrm{P}, 0.021 ; \mathrm{S}, 0.010 ; \mathrm{Si}, 0.20 ; \mathrm{Ni}, 3.43$ ]

\begin{tabular}{|c|c|c|c|c|c|c|}
\hline Number & $\underset{\text { test }}{\text { Temperature of }}$ & $\begin{array}{l}\text { Propor- } \\
\text { tiona 1 } \\
\text { limit }\end{array}$ & $\begin{array}{l}\text { Tensile } \\
\text { strength }\end{array}$ & $\begin{array}{l}\text { Breaking } \\
\text { strength } a\end{array}$ & $\begin{array}{l}\text { Elonga- } \\
\text { tion in } \\
2 \text { inches }\end{array}$ & $\begin{array}{l}\text { Reduc- } \\
\text { tion of } \\
\text { area }\end{array}$ \\
\hline 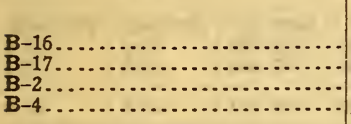 & $\{(b) \ldots \ldots \ldots \ldots$ & $\left\{\begin{array}{c}\text { Lbs./in. }{ }^{2} \\
57000 \\
56000 \\
52500\end{array}\right.$ & $\begin{array}{r}\text { Lbs./in. }{ }^{2} \\
105150 \\
104900 \\
103600 \\
103400\end{array}$ & $\begin{array}{r}\text { Lbs./in.2 } \\
172500 \\
164000 \\
152000 \\
170800\end{array}$ & \begin{tabular}{|r|} 
Per cent \\
30.5 \\
27.5 \\
27.5 \\
28.0
\end{tabular} & $\begin{array}{r}\text { Per cent } \\
50.6 \\
46.7 \\
39.9 \\
49.7\end{array}$ \\
\hline Average................... & $21^{\circ} \mathrm{C}\left(70^{\circ} \mathrm{F}\right) \ldots$ & 51850 & 104250 & 164800 & 28.4 & 46.7 \\
\hline $\begin{array}{l}\text { B-21 } \\
\text { B-22 } \\
\text { B-1 } \\
\text { B-3 }\end{array}$ & $\begin{array}{l}92^{\circ} \mathrm{C} \ldots \ldots \ldots \ldots \\
92^{\circ} \mathrm{C} \ldots \ldots \ldots \ldots \ldots \\
92^{\circ} \mathrm{C} \ldots \ldots \ldots \ldots \ldots \\
92^{\circ} \mathrm{C} \ldots \ldots \ldots \ldots \\
\end{array}$ & $\begin{array}{r}53000 \\
58000 \\
\cdots\end{array}$ & $\begin{array}{ll}97 & 600 \\
97 & 800 \\
99 & 600 \\
99 & 300\end{array}$ & $\begin{array}{l}169200 \\
170300 \\
169500 \\
172300\end{array}$ & $\begin{array}{l}28.5 \\
29.0 \\
26.0 \\
28.0\end{array}$ & $\begin{array}{l}54.1 \\
52.3 \\
51.3 \\
52.4\end{array}$ \\
\hline Average .............. & $92^{\circ} \mathrm{C}\left(198^{\circ} \overline{\mathrm{F}}\right)$ & 55500 & 98600 & 170300 & 27.9 & 52.5 \\
\hline 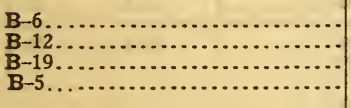 & $\begin{array}{l}155^{\circ} \mathrm{C} \ldots \ldots \ldots \ldots \\
155^{\circ} \mathrm{C} \ldots \ldots \ldots \ldots \\
155^{\circ} \mathrm{C} \ldots \ldots \ldots \ldots \ldots \\
155^{\circ} \mathrm{C} \ldots \ldots \ldots \ldots \\
\end{array}$ & $\begin{array}{r}52500 \\
56000 \\
55000 \\
\end{array}$ & $\begin{array}{ll}97 & 100 \\
96 & 200 \\
98 & 300 \\
95 & 000 \\
\end{array}$ & $\begin{array}{l}170800 \\
159700 \\
169300 \\
161300\end{array}$ & $\begin{array}{l}22.0 \\
24.5 \\
25.0 \\
23.5\end{array}$ & $\begin{array}{l}43.2 \\
47.3 \\
49.4 \\
48.5\end{array}$ \\
\hline Average.. & $155^{\circ} \mathrm{C}\left(311^{\circ} \mathrm{F}\right) \ldots$ & 54500 & 96650 & 165300 & 23.8 & 47.1 \\
\hline $\begin{array}{l}\text { B-18.. } \\
\text { B-7... } \\
\text { B-8... }\end{array}$ & $\begin{array}{l}241^{\circ} \mathrm{C} \ldots \ldots \ldots \\
241^{\circ} \mathrm{C} \ldots \ldots \ldots \\
241^{\circ} \mathrm{C} \ldots \ldots \ldots\end{array}$ & $\begin{array}{r}42500 \\
42500 \\
\end{array}$ & $\begin{array}{l}104600 \\
106200 \\
103500\end{array}$ & $\begin{array}{ll}181200 \\
163600 \\
154300\end{array}$ & $\begin{array}{l}25.0 \\
22.0 \\
21.5\end{array}$ & $\begin{array}{l}51.5 \\
40.6 \\
40.9\end{array}$ \\
\hline Average. & $241^{\circ} \mathrm{C}\left(466^{\circ} \mathrm{F}\right) \ldots$ & 42500 & 104750 & 166350 & 22.8 & 44.3 \\
\hline $\begin{array}{l}\text { B-10..... } \\
\text { B-9..... }\end{array}$ & $\begin{array}{l}294^{\circ} \mathrm{C} \ldots \ldots \ldots \\
294^{\circ} \mathrm{C} \ldots \ldots \ldots\end{array}$ & $\begin{array}{l}34000 \\
36500 \\
\end{array}$ & $\begin{array}{l}109500 \\
108900 \\
\end{array}$ & $\begin{array}{l}194400 \\
194800 \\
\end{array}$ & $\begin{array}{l}31.0 \\
32.0\end{array}$ & $\begin{array}{l}60.2 \\
59.7\end{array}$ \\
\hline Average. & $294^{\circ} \mathrm{C}\left(561^{\circ} \mathrm{F}\right) \ldots$ & 35250 & 109200 & 194600 & 31.5 & 60.0 \\
\hline $\begin{array}{l}\text { B-15. } \\
\text { B-13. }\end{array}$ & $\begin{array}{l}407^{\circ} \mathrm{C} \ldots \ldots \\
407^{\circ} \mathrm{C} \ldots \ldots\end{array}$ & $\begin{array}{ll}21500 \\
24000 \\
\end{array}$ & $\begin{array}{l}83000 \\
83250 \\
\end{array}$ & $\begin{array}{l}152600 \\
169700 \\
\end{array}$ & $\begin{array}{l}31.5 \\
31.5 \\
\end{array}$ & $\begin{array}{l}74.4 \\
74.5 \\
\end{array}$ \\
\hline Average.. & $407^{\circ} \mathrm{C}\left(765^{\circ} \mathrm{F}\right) \ldots$ & 22750 & 83150 & 161150 & 31.5 & 74.5 \\
\hline B-14............. & $\begin{array}{l}453^{\circ} \mathrm{C} \ldots \ldots . \\
463^{\circ} \mathrm{C} . \ldots .\end{array}$ & $\begin{array}{l}16000 \\
18500 \\
\end{array}$ & $\begin{array}{l}63900 \\
63500\end{array}$ & $\begin{array}{l}126400 \\
127900\end{array}$ & $\begin{array}{l}35.0 \\
35.0 \\
\end{array}$ & $\begin{array}{l}79.6 \\
79.7 \\
\end{array}$ \\
\hline Average. . & $463^{\circ} \mathrm{C}\left(865^{\circ} \mathrm{F}\right) \ldots$ & 17250 & 63700 & 127150 & 35.0 & 79.7 \\
\hline $\begin{array}{l}\mathrm{B}-24 \ldots \ldots \\
\mathrm{B}-25 \ldots\end{array}$ & $\begin{array}{l}550^{\circ} \mathrm{C} \ldots \ldots . \\
550^{\circ} \mathrm{C} . \ldots . . .\end{array}$ & $\begin{array}{l}7500 \\
9000 \\
\end{array}$ & $\begin{array}{ll}37400 \\
33 & 300 \\
\end{array}$ & $\begin{array}{l}79000 \\
76300 \\
\end{array}$ & $\begin{array}{l}42.5 \\
44.5 \\
\end{array}$ & $\begin{array}{l}88.3 \\
89.5 \\
\end{array}$ \\
\hline Average. & $550^{\circ} \mathrm{C}\left(1022^{\circ} \mathrm{F}\right) \ldots$ & 8250 & 35350 & 77650 & 43.5 & 88.9 \\
\hline
\end{tabular}

a Breaking strength is taken as the load observed at fracture (in pounds) divided by the reduced area (in square inches).

boom temperature. 
TABLE 6.-Tensile Properties at Elevated Temperatures of Nickel-Chromium Steel of the Type 3 Per Cent Nickel, 1 Per Cent Chromium, 0.4 Per Cent Carbon

[Chemical composition (per cent): C, 0.39; $\mathrm{Mn}, 0.59 ; \mathrm{P}, 0.019 ; \mathrm{S}, 0.009 ; \mathrm{Si}, 0.23 ; \mathrm{Ni}, 3.05 ; \mathrm{Cr}, 0.93$ ]

\begin{tabular}{|c|c|c|c|c|c|c|}
\hline Number & $\begin{array}{c}\text { Temperature of } \\
\text { test }\end{array}$ & $\begin{array}{l}\text { Propor- } \\
\text { tional } \\
\text { limit }\end{array}$ & $\begin{array}{l}\text { Tensile } \\
\text { strength }\end{array}$ & $\begin{array}{l}\text { Breaking } \\
\text { strength } a\end{array}$ & $\begin{array}{l}\text { Elonga- } \\
\text { tion in } \\
2 \text { inches }\end{array}$ & $\begin{array}{l}\text { Reduc- } \\
\text { tion of } \\
\text { area }\end{array}$ \\
\hline$\stackrel{\mathrm{C}-14}{\mathrm{C}-15}$ & \}$(b)$ & $\left\{\begin{array}{c}\text { Lbs./in. }{ }^{2} \\
58500 \\
58500\end{array}\right.$ & \begin{tabular}{|c|} 
Lbs./in. ${ }^{2}$ \\
175000 \\
171800
\end{tabular} & \begin{tabular}{|c|} 
Lbs./in. \\
213 \\
207000 \\
207000
\end{tabular} & $\begin{array}{r}\text { Per cen } t \\
10.0 \\
11.0\end{array}$ & $\begin{array}{r}\text { Per cent } \\
22.9 \\
25.2\end{array}$ \\
\hline Average. & $21^{\circ} \mathrm{C}\left(70^{\circ} \mathrm{F}\right) \ldots$ & 58500 & 173400 & 210350 & 10.5 & 24.0 \\
\hline$\stackrel{C}{\mathrm{C}-2} \ldots \ldots \ldots$ & $\begin{array}{l}92^{\circ} \mathrm{C} \ldots \ldots \ldots \\
92^{\circ} \mathrm{C} \ldots \ldots \ldots \ldots\end{array}$ & $\begin{array}{ll}84 & 700 \\
74 & 000\end{array}$ & $\begin{array}{l}175000 \\
170000\end{array}$ & $\cdots$ & $\begin{array}{l}11.0 \\
11.0\end{array}$ & $\begin{array}{l}20.3 \\
23.1 \\
\end{array}$ \\
\hline Average. & $92^{\circ} \mathrm{C}\left(198^{\circ} \mathrm{F}\right)$. & 79350 & 172500 & $\cdots$ & 11.0 & 21.7 \\
\hline $\begin{array}{l}\mathbf{C}-6 \ldots \ldots \ldots \ldots \\
\mathbf{C}-7 . \ldots \ldots \ldots\end{array}$ & $\begin{array}{l}155^{\circ} \mathrm{C} \ldots \ldots \ldots \\
155^{\circ} \mathrm{C} \ldots \ldots \ldots\end{array}$ & $\begin{array}{ll}75 & 000 \\
72 & 500\end{array}$ & $\begin{array}{l}175200 \\
166000\end{array}$ & $\cdots$ & $\begin{array}{l}12.5 \\
12.5\end{array}$ & $\begin{array}{l}23.2 \\
26.2\end{array}$ \\
\hline Average .. & $155^{\circ} \mathrm{C}\left(311^{\circ} \mathrm{F}\right)$. & 73750 & 170600 & $\ldots$ & 12.5 & 24.7 \\
\hline $\begin{array}{l}\mathrm{C}-4 \\
\mathrm{C}-5\end{array}$ & $\begin{array}{l}241^{\circ} \mathrm{C} . \ldots \ldots \ldots \\
241^{\circ} \mathrm{C} \ldots \ldots \ldots \ldots\end{array}$ & $\begin{array}{ll}63 & 500 \\
62 & 500 \\
\end{array}$ & $\begin{array}{ll}181300 \\
178000 \\
\end{array}$ & $\ddot{-}$ & $\begin{array}{c}18.0 \\
\ldots \ldots \ldots \\
\end{array}$ & $\begin{array}{l}39.6 \\
\cdots \cdots \\
\end{array}$ \\
\hline Average & $241^{\circ} \mathrm{C}\left(466^{\circ} \mathrm{F}\right)$ & 63000 & 179650 & $=$ & & \\
\hline 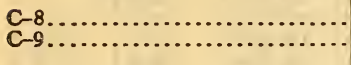 & $\begin{array}{l}294^{\circ} \mathrm{C} \ldots \ldots \ldots \ldots \\
294^{\circ} \mathrm{C} \ldots \ldots \ldots \ldots \ldots\end{array}$ & $\begin{array}{l}75000 \\
80000 \\
\end{array}$ & $\begin{array}{l}174000 \\
171000 \\
\end{array}$ & a... & $\begin{array}{l}23.5 \\
23.5\end{array}$ & $\begin{array}{l}62.4 \\
63.8 \\
\end{array}$ \\
\hline Average.. & $294^{\circ} \mathrm{C}\left(561^{\circ} \mathrm{F}\right) \ldots$ & 77500 & 172500 & $\ldots$ & 23.5 & 63.1 \\
\hline $\begin{array}{l}\mathbf{C}-10 \ldots \ldots \\
\mathbf{C}-11 \ldots \ldots\end{array}$ & $\begin{array}{l}407^{\circ} \mathrm{C} \ldots \ldots \ldots \ldots \\
497^{\circ} \mathrm{C} . \ldots \ldots \ldots\end{array}$ & $\begin{array}{ll}43000 \\
49000 \\
\end{array}$ & $\begin{array}{ll}127600 \\
132 & 200 \\
\end{array}$ & $\begin{array}{ll}181200 \\
209000\end{array}$ & $\begin{array}{l}20.0 \\
20.0\end{array}$ & $\begin{array}{l}66.5 \\
65.4 \\
\end{array}$ \\
\hline Average. . & $407^{\circ} \mathrm{C}\left(765^{\circ} \mathrm{F}\right)$ & 46000 & 129900 & 195100 & 20.0 & 66.0 \\
\hline $\begin{array}{l}\mathrm{C}-12 \ldots \\
\mathrm{C}-13 \ldots\end{array}$ & $\begin{array}{l}463^{\circ} \mathrm{C} \ldots \ldots \ldots \\
463^{\circ} \mathrm{C} . \ldots \ldots\end{array}$ & $\begin{array}{ll}22000 \\
21500 \\
\end{array}$ & $\begin{array}{l}105000 \\
102500 \\
\end{array}$ & $\begin{array}{l}137700 \\
150300 \\
\end{array}$ & $\begin{array}{l}19.0 \\
19.0 \\
\end{array}$ & $\begin{array}{l}59.9 \\
62.0 \\
\end{array}$ \\
\hline Average. & $463^{\circ} \mathrm{C}\left(865^{\circ} \mathrm{F}\right)$. & 21750 & 103750 & 144000 & 19.0 & 61.0 \\
\hline $\begin{array}{l}\mathrm{C}-16 . \\
\mathrm{C}-17 .\end{array}$ & $\begin{array}{l}550^{\circ} \mathrm{C} \ldots \ldots \\
550^{\circ} \mathrm{C} . . .\end{array}$ & $\begin{array}{l}12000 \\
15000\end{array}$ & $\begin{array}{ll}72 & 100 \\
69 & 500\end{array}$ & $\begin{array}{ll}90 & 000 \\
95 & 750\end{array}$ & $\begin{array}{l}19.0 \\
22.5\end{array}$ & $\begin{array}{l}59.0 \\
67.3\end{array}$ \\
\hline Average... & $550^{\circ} \mathrm{C}\left(1022^{\circ} \bar{F}\right) \ldots$ & 13500 & 70800 & 92850 & 20.8 & 63.2 \\
\hline
\end{tabular}

a Breaking strength is taken as the load observed at fracture (in pounds) divided by the reduced area (in square inches).

Room temperature. 
TABLE 7.-Tensile Properties at Elevated Temperatures of Chromium-Vanadium Steel of the Type 1 Per Cent Chromium, 0.2 Per Cent Vanadium, 0.37 Per Cent Carbon

[Chemical composition (per cent): C, 0.37; $\mathrm{Mn}, 0.74 ; \mathrm{P}, 0.020 ; \mathrm{S}, 0.023 ; \mathrm{Si}, 0.21 ; \mathrm{Cr}, 1.04 ; \mathrm{V}, 0.17$ ]

\begin{tabular}{|c|c|c|c|c|c|c|}
\hline Number & $\begin{array}{c}\text { Temperature of } \\
\text { test }\end{array}$ & $\begin{array}{l}\text { Propor- } \\
\text { tional } \\
\text { limit }\end{array}$ & $\begin{array}{l}\text { Tensile } \\
\text { strength }\end{array}$ & $\begin{array}{l}\text { Breaking } \\
\text { strengtha }\end{array}$ & $\begin{array}{l}\text { Elonga- } \\
\text { tion in } \\
2 \text { inches }\end{array}$ & $\begin{array}{l}\text { Reduc- } \\
\text { tion of } \\
\text { area }\end{array}$ \\
\hline $\begin{array}{l}\text { D1 } \ldots \ldots . . . \\
\text { D-16..... }\end{array}$ & \} (b). & $\left\{\begin{array}{c}\text { Lbs./in. } .^{2} \\
88000 \\
88000\end{array}\right.$ & $\begin{array}{r}\text { Lbs./in. }{ }^{2} \\
141600 \\
147000\end{array}$ & $\begin{array}{c}\text { Ibs./in. } .^{2} \\
236000 \\
224500\end{array}$ & $\begin{array}{r}\text { Per cent } \\
20.0 \\
19.0\end{array}$ & $\begin{array}{r}\text { Per cent } \\
55.5 \\
49.4\end{array}$ \\
\hline Average. & $21^{\circ} \mathrm{C}\left(70^{\circ} \mathrm{F}\right)$. & 88000 & 144300 & 230250 & 19.5 & 42.5 \\
\hline $\begin{array}{l}\text { D-3........ } \\
\text { D-4....... } \\
\text { D-15...... }\end{array}$ & 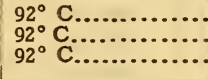 & $\begin{array}{ll}81 & 000 \\
70 & 000 \\
73 & 000\end{array}$ & $\begin{array}{l}150800 \\
135100 \\
139500\end{array}$ & $\begin{array}{ll}229 & 400 \\
224 & 300 \\
224 & 100\end{array}$ & $\begin{array}{l}17.5 \\
18.0 \\
17.5\end{array}$ & $\begin{array}{l}48.4 \\
51.7 \\
46.2\end{array}$ \\
\hline Average. & $92^{\circ} \mathrm{C}\left(198^{\circ} \mathrm{F}\right)$ & 74650 & 141800 & 225950 & 17.7 & 48.8 \\
\hline $\begin{array}{l}\text { D-7......... } \\
\text { D-8........ }\end{array}$ & $\begin{array}{l}155^{\circ} \mathrm{C} \ldots \ldots \ldots \\
155^{\circ} \mathrm{C} \ldots \ldots \ldots\end{array}$ & $\begin{array}{l}76000 \\
79000 \\
\end{array}$ & $\begin{array}{l}136200 . \\
146500 \\
\end{array}$ & $\begin{array}{l}240000 \\
203000 \\
\end{array}$ & $\begin{array}{l}20.0 \\
\cdots \cdots \\
\end{array}$ & $\begin{array}{r}55.7 \\
\cdots \cdots \\
\end{array}$ \\
\hline Averag & $155^{\circ} \mathrm{C}\left(311^{\circ} \mathrm{F}\right)$ & 77500 & 141350 & 221500 & $\cdots$ & $\cdots$ \\
\hline $\begin{array}{l}D-5 \ldots . \\
D-6 \ldots\end{array}$ & $241^{\circ} \mathrm{C} \ldots \ldots . .$. & $\begin{array}{l}81000 \\
80000\end{array}$ & $\begin{array}{l}133600 \\
137200\end{array}$ & $\begin{array}{l}252300 \\
238700\end{array}$ & $\begin{array}{l}21.0 \\
19.0\end{array}$ & $\begin{array}{l}58.6 \\
53.3\end{array}$ \\
\hline Average. & $241^{\circ} \mathrm{C}\left(466^{\circ} \mathrm{F}\right) \ldots$ & 80500 & 135400 & 245500 & 20.0 & 56.0 \\
\hline $\begin{array}{l}D-9 \ldots \ldots \ldots \\
D-10 \ldots \ldots . .\end{array}$ & $294^{\circ} \mathrm{C} \ldots \ldots \ldots . .$. & $\begin{array}{l}76000 \\
73000\end{array}$ & $\begin{array}{l}145500 \\
139000\end{array}$ & $\begin{array}{ll}223 & 400 \\
261 & 300\end{array}$ & $\begin{array}{r}19.0 \\
\ldots \ldots \ldots\end{array}$ & $\begin{array}{r}45.8 \\
\ldots \ldots .6 .\end{array}$ \\
\hline Average. & $294^{\circ} \mathrm{C}\left(561^{\circ} \mathrm{F}\right) \ldots$ & 74500 & 142250 & 242350 & $\ldots \ldots \ldots$ & ............ \\
\hline $\begin{array}{l}\text { D-11........ } \\
\text { D-12....... }\end{array}$ & $\begin{array}{l}407^{\circ} \mathrm{C} \ldots \ldots \ldots \ldots \ldots \\
407^{\circ} \mathrm{C} \ldots \ldots \ldots \ldots \ldots\end{array}$ & $\begin{array}{ll}50 & 000 \\
51 & 500\end{array}$ & $\begin{array}{l}108000 \\
115200 \\
\end{array}$ & $\begin{array}{l}182300 \\
213000\end{array}$ & $\begin{array}{l}25.0 \\
25.0\end{array}$ & $\begin{array}{l}67.7 \\
71.2\end{array}$ \\
\hline Average. & $407^{\circ} \mathrm{C}\left(765^{\circ} \mathrm{F}\right) \ldots$ & 50750 & 111600 & 197500 & 25.0 & 69.5 \\
\hline $\begin{array}{l}\text { D }-13 \ldots \ldots \ldots . . . \\
D-14 \ldots \ldots \ldots . . .\end{array}$ & $463^{\circ} \mathrm{C} \ldots \ldots \ldots . . . .$. & $\begin{array}{l}27000 \\
29000 \\
\end{array}$ & $\begin{array}{r}97500 \\
101000 \\
\end{array}$ & $\begin{array}{l}122500 \\
147300 \\
\end{array}$ & $\begin{array}{l}23.5 \\
22.5 \\
\end{array}$ & $\begin{array}{l}52.9 \\
62.4 \\
\end{array}$ \\
\hline Average. & $463^{\circ} \mathrm{C}\left(865^{\circ} \mathrm{F}\right)$ & 28000 & 99250 & 134900 & 23.0 & 57.7 \\
\hline $\begin{array}{l}\text { D-18... } \\
\text { D-19... }\end{array}$ & $550^{\circ} \mathrm{C} \ldots \ldots$. & $\begin{array}{l}16500 \\
17500\end{array}$ & $\begin{array}{l}82600 \\
84000\end{array}$ & $\begin{array}{l}118800 \\
111000\end{array}$ & $\begin{array}{l}22.0 \\
19.0\end{array}$ & $\begin{array}{l}61.7 \\
54.4\end{array}$ \\
\hline Average. & $550^{\circ} \mathrm{C}\left(1022^{\circ} \mathrm{F}\right) \ldots$ & 17000 & 83300 & 114900 & 20.5 & 58.0 \\
\hline
\end{tabular}

a Breaking strength is taken as the load observed at fracture (in pounds) divided by the reduced area (in square inches).

$b$ Room temperature.

Maximum strength of the carbon, $3 \frac{1}{2}$ per cent nickel and nickelchromium steels, occurs between about 240 and $300^{\circ} \mathrm{C}(465$ and $565^{\circ} \mathrm{F}$ ), whereas the strength of the chromium-vanadium steel does not exceed its room temperature value within the temperature range under consideration. However, a rapid decrease in this factor is observed in all steels above $300^{\circ} \mathrm{C}\left(565^{\circ} \mathrm{F}\right)$.

At $550^{\circ} \mathrm{C}\left(1020^{\circ} \mathrm{F}\right)$ the strength of the chromium-vanadium steel is very much greater than that of the other alloys and more than twice that of the carbon steel. It has likewise decreased the least in strength from its room temperature value, as shown in Table 8. However, at all temperatures below about $475^{\circ} \mathrm{C}$ $\left(885^{\circ} \mathrm{F}\right)$ the strength of the nickel-chromium steel is greater than that of the chromium-vanadium. 
TABLE 8.-Comparison of Tensile Strengths of Carbon and Alloy Steels at Room Temperature and $550^{\circ} \mathrm{C}$

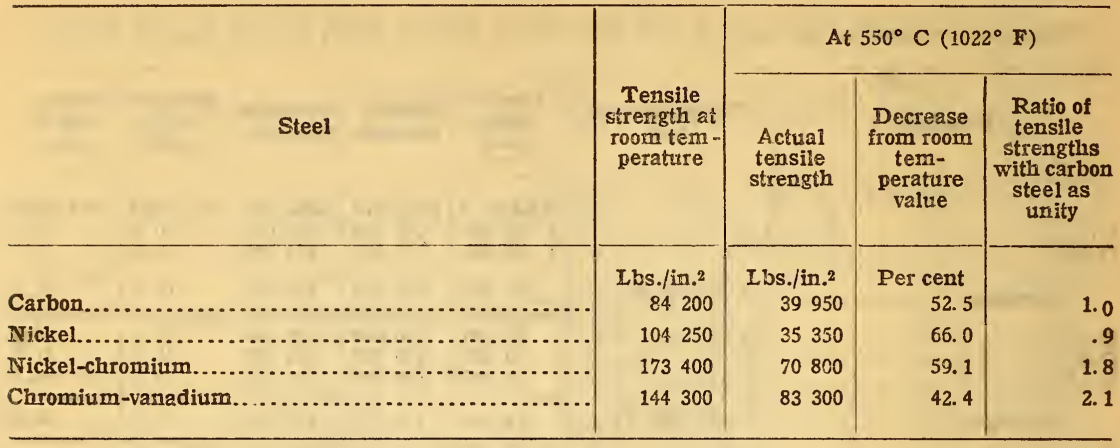

(b) PROPORTIONAL LIMIT.-The proportional limits of the carbon, $3 \mathrm{I} / 2$ per cent nickel and nickel-chromium steels, increase with first rise in temperature and reach maximum values in the neighborhood of $150^{\circ} \mathrm{C}\left(300^{\circ} \mathrm{F}\right)$, whereas that of the chromiumvanadium steel is greatest at room temperature and, following a marked decrease, again increases perceptibly between about I5O to $250^{\circ} \mathrm{C}\left(300\right.$ to $\left.480^{\circ} \mathrm{F}\right)$ before final decrease occurs. While the maximum value of the limit of proportionality of the nickelchromium steel occurs at about $150^{\circ} \mathrm{C}\left(300^{\circ} \mathrm{F}\right)$ and is followed by a material decrease, a second rise in value is observed at about $300^{\circ} \mathrm{C}\left(570^{\circ} \mathrm{F}\right)$, so that this factor is maintained above its room temperature value until temperatures above about $370^{\circ} \mathrm{C}$ $\left(700^{\circ} \mathrm{F}\right)$ are reached.

The limit of proportionality of the chromium-vanadium steel at $550^{\circ} \mathrm{C}\left(1 \mathrm{O} 20^{\circ} \mathrm{F}\right.$ ) has decreased proportionally more than either the carbon or nickel-chromium steels, as shown in Table 9, but its value at the high temperature indicated is greater than either of the latter and more than twice that of the carbon steel.

FABLE 9.-Comparison of Proportional Limits of Carbon and Alloy Steels at Room Temperature and $550^{\circ} \mathrm{C}$

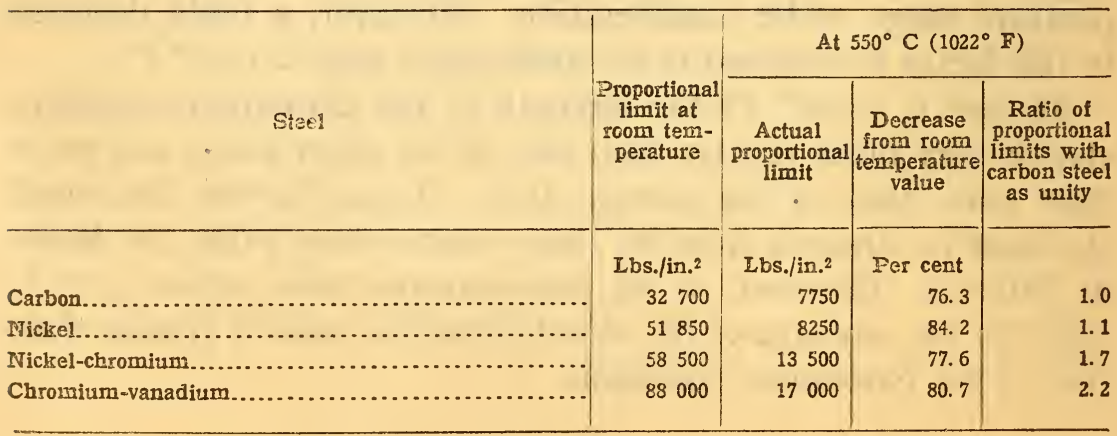


(c) Elongation and REDUCTION OF AREA.-The effect of temperature is greater upon the values of reduction of area than elongation. In general, the inflections in the temperature elongation and temperature reduction of area curves shown in Figs. $2,3,4$, and 5 are so varied that no attempt will be made to describe them. However, several generalizations may be made. The carbon and $3 \frac{1}{2}$ per cent nickel steels behave similarly, in that rise in temperature is accompanied by a general decrease in ductility, which is followed above the range 200 to $300^{\circ} \mathrm{C}\left(390\right.$ to $\left.565^{\circ} \mathrm{F}\right)$ by an increase until at $550^{\circ} \mathrm{C}\left(1020^{\circ} \mathrm{F}\right)$ values of elongation and reduction of area are greater than those observed at room temperature.

The elongation of the chromium-vanadium steel is about the same at $55^{\circ} \mathrm{C}\left(1020^{\circ} \mathrm{F}\right)$ as at room temperature and does not show any very great changes throughout this temperature range, whereas elongation of the nickel-chromium steel increases to a maximum at about $295^{\circ} \mathrm{C}\left(565^{\circ} \mathrm{F}\right)$ (blue heat).

While the carbon and nickel steels are much more ductile at $550^{\circ} \mathrm{C}\left(1020^{\circ} \mathrm{F}\right)$ than either the chromium-vanadium or nickelchromium steels, as shown in Tables ro and II, yet the last two alloys have high ductility, and the latter shows by far the greatest increase over its room temperature value.

TABLE 10.-Comparison of Elongations of Carbon and Alloy Steels at Room Temperature and $550^{\circ} \mathrm{C}$

\begin{tabular}{|c|c|c|c|c|c|}
\hline & \multirow[b]{2}{*}{ Stecl } & \multirow[b]{2}{*}{$\begin{array}{c}\text { Elongation } \\
\text { in } 2 \text { inches } \\
\text { at room } \\
\text { temperature }\end{array}$} & \multicolumn{3}{|c|}{ At $550^{\circ} \mathrm{C}\left(1022^{\circ} \mathrm{F}\right)$} \\
\hline- & & & $\begin{array}{c}\text { Actual } \\
\text { elongation } \\
\text { in } 2 \text { inches }\end{array}$ & $\mid \begin{array}{c}\text { Increase } \\
\text { from room } \\
\text { temperature } \\
\text { value }\end{array}$ & $\begin{array}{l}\text { Ratio of } \\
\text { elongations } \\
\text { with carbon } \\
\text { steel as } \\
\text { unity }\end{array}$ \\
\hline \multicolumn{2}{|l|}{ Carbon.. } & $\begin{array}{r}\text { Per cent } \\
30.5\end{array}$ & $\begin{array}{r}\text { Per cent } \\
40.5\end{array}$ & $\begin{array}{r}\text { Per cent } \\
32.8\end{array}$ & 1.0 \\
\hline \multicolumn{2}{|l|}{ Nickel... } & 28.4 & 43.5 & 51.4 & 1.1 \\
\hline \multicolumn{2}{|c|}{ 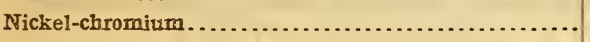 } & 10.5 & 20.8 & 98.0 & .5 \\
\hline \multicolumn{2}{|c|}{ 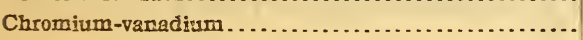 } & 19.5 & 20.5 & 5.1 & .5 \\
\hline
\end{tabular}

TABLE 11. Comparison of Reductions of Area of Carbon and Alloy Steels at Roon Temperature and $550^{\circ} \mathrm{C}$

\begin{tabular}{|c|c|c|c|c|}
\hline 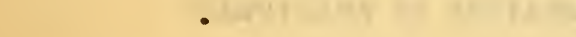 & & & $550^{\circ} \mathrm{C}(1022$ & $\left.2^{\circ} \mathrm{F}\right)$ \\
\hline Stcel & $\begin{array}{c}\text { Reduction } \\
\text { of area } \\
\text { at room } \\
\text { temperature }\end{array}$ & $\begin{array}{c}\text { Actual } \\
\text { reduction } \\
\text { of area }\end{array}$ & $\begin{array}{c}\text { Increase } \\
\text { from room } \\
\text { temperature } \\
\text { vaiue }\end{array}$ & $\begin{array}{c}\text { Ratio of } \\
\text { redictions } \\
\text { of area } \\
\text { with carbon } \\
\text { steel as } \\
\text { unity }\end{array}$ \\
\hline Carbon.. & $\begin{array}{r}\text { Per cent } \\
52.8\end{array}$ & $\begin{array}{r}\text { Per cent } \\
92.7\end{array}$ & $\begin{array}{r}\text { Per cent } \\
75.5\end{array}$ & 1.0 \\
\hline Nickel. & 46.7 & 88.9 & 90.3 & 1.0 \\
\hline Niefel-chromium.. & 24.0 & 63.2 & 163.3 & .7 \\
\hline Chromilum-vanadium. & 42.5 & 58.0 & 36.5 & .6 \\
\hline
\end{tabular}


(d) BREAKING STRENGTH.-The strength at fracture of the different steels tested, obtained by dividing the load observed at the moment of breaking by the reduced area, is shown in Fig. 6 . While the data are incomplete and in some cases the variations in duplicate determinations are wide, the results are interesting when taken in conjunction with the changes previously described, for at $550^{\circ} \mathrm{C}\left(\mathrm{IO}_{2} \mathrm{O}^{\circ} \mathrm{F}\right)$ the highest breaking strength is shown

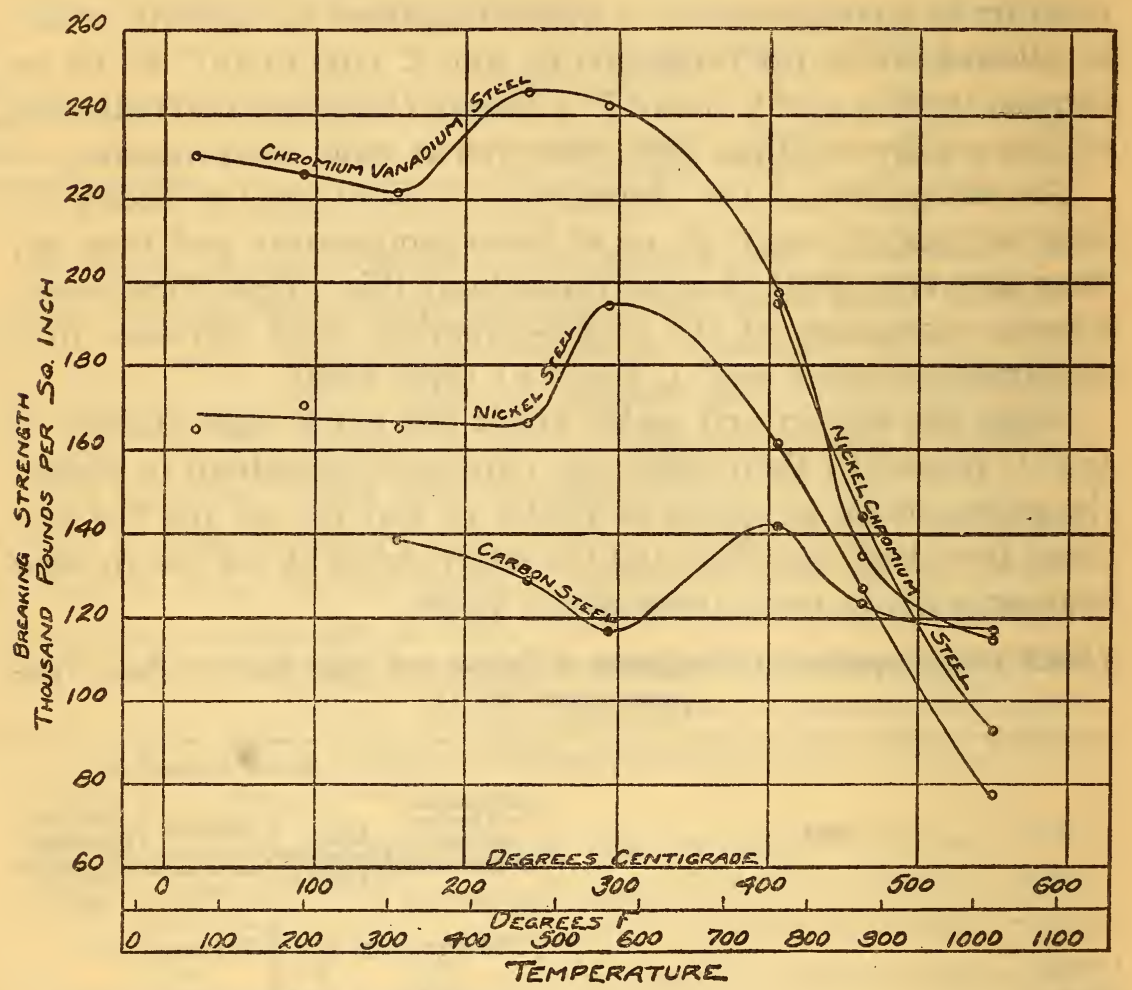

Fi.G. 6.-Breaking strength at elevated temperatures of carbon, nickel, nickel-chromium, and chromium-vanadium steels. (For compositions refer to Table 3)

by the chromium-vanadium and carbon steels and the lowest value is observed in the alloy containing $3 \frac{1}{2}$ per cent nickel.

\section{MICROSCOPIC EXAMINATION OF FRACTURES}

The fracture of steels subjected to tensile stress at room temperature is transcrystalline, while at high temperatures it takes place at the grain boundaries. The change from transcrystalline to intercrystalline fractures will occur beginning at temperatures somewhat above that of equal cohesion of the amorphous and crystalline phases, but the temperatures at which these fractures 


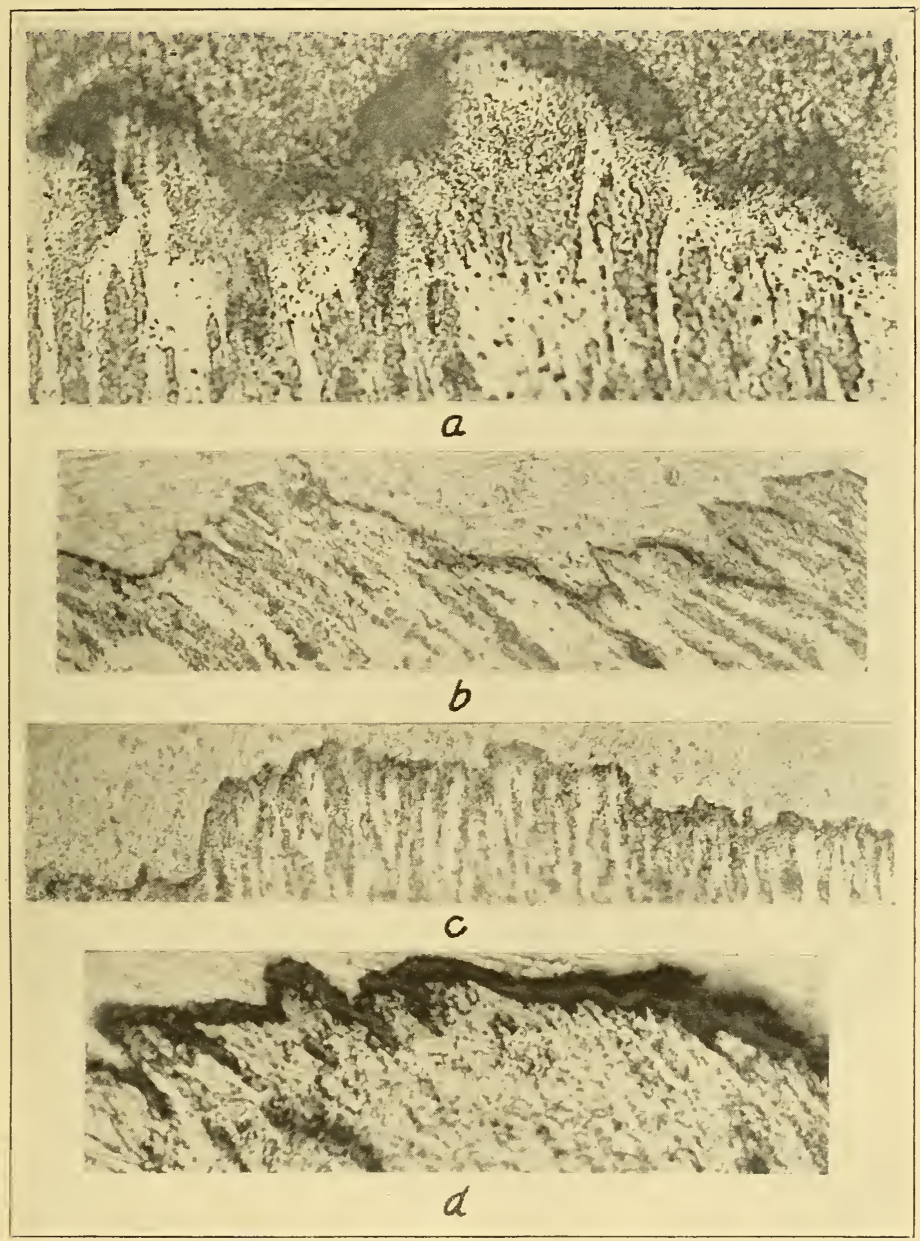

FIG. 7.-Microphotographs of fractures of carbon and $3 \mathrm{I} / 2$ per cent nickel steels broken in tension at high temperatures
(a) Specimen A I5. Broken at $453^{\circ} \mathrm{C} . \quad \times_{500}$
(b) Specimen $\mathrm{A}_{22}$. Broken at $550^{\circ} \mathrm{C}$. $X_{500}$
(c) Specimen B Ir. Broken at $463^{\circ} \mathrm{C}$. $\times_{500}$
(d) Specimen $\mathrm{B} 24$. Broken at $550^{\circ} \mathrm{C}$. $\times_{1000}$
All specinens etched with 5 per cent picric acid in alcohoi 
Technologic Papers of the Bureau of Standards, Vol. 16

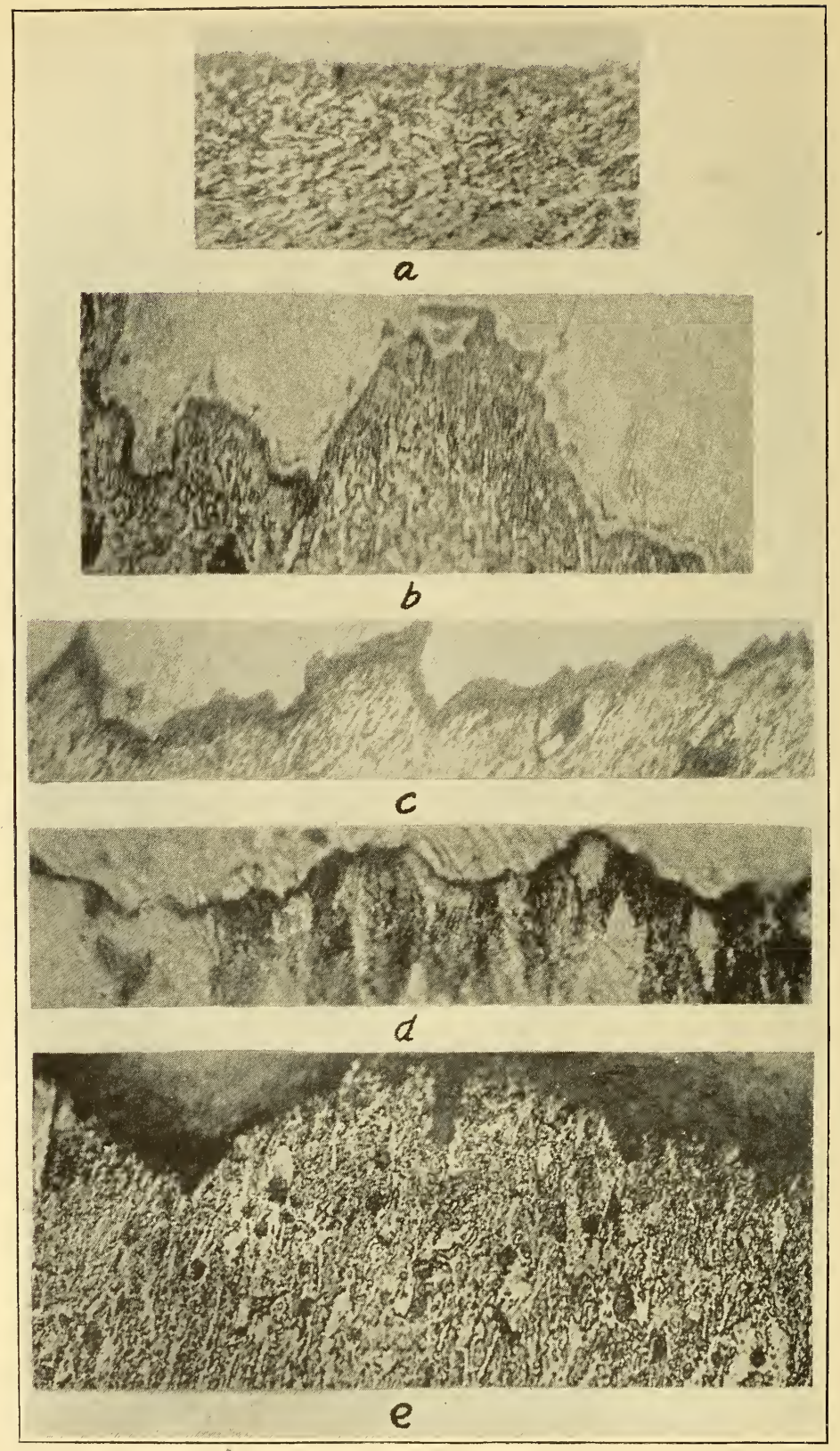

Fig. 8.-Microphotographs of fractures of nickel-chromium and chromium-vanadium steels broken in tension at high temperatures

(a) Specimen C 8. Broken at $294^{\circ} \mathrm{C} . \times_{1000}$

(b) Specimen $\mathrm{C}_{\mathrm{I}_{3}}$. Broken at $463^{\circ} \mathrm{C} . \mathrm{X}_{500}$

(c) Specimen C r6: Broken at $550^{\circ} \mathrm{C}$. $\times_{500}$

(d) Specimen D r 4 . Broken at $463^{\circ} \mathrm{C}$. $\times$ rooo

(e) Specimen D r8. Broken at $550^{\circ} \mathrm{C}$. $\times_{500}$

$a, d$, and $e$ etched with 5 per cent picric acid in alcohol; $b$ and $c$ with 2 per cent nitric acid in alcohol 
first appear in a given alloy depend upon the rate of loading. 9 The faster the load is applied the higher is the temperature required to produce intercrystalline breaks.

Jeffries states that in most metals the equicohesive temperature occurs at about 0.35 to 0.45 of the melting point on the absolute temperature scale, so that for steels the change from fractures across the grains to those along the crystal boundaries should on slow loading first appear in the neighborhood of $550^{\circ} \mathrm{C}$ (Iо20 ${ }^{\circ} \mathrm{F}$ ). This temperature is approximately the highest at which tensile tests were made, and, as these were likewise considered of particular interest from the standpoint of operation in the Haber process, specimens of the several steels tested were examined under the microscope to determine the character of the fractures.

Typical microphotographs are reproduced in Figs. 7 and 8 , and while no general statements can be made with certainty they show the tendency of the fracture in the carbon and $3 \frac{1}{2}$ per cent nickel steels to follow the grain boundaries when broken at about $550^{\circ} \mathrm{C}\left(1020^{\circ} \mathrm{F}\right)$.

The outstanding feature of the nickel-chromium steel is the fineness of the structure, making it difficult to determine the character of the break, while in the case of the chromium-vanadium steel the fractures appear more nearly intracrystalline (8e) even at the highest temperatures under investigation.

\section{SUMMARY}

The following points appear to deserve emphasis in connection with the tests previously described:

I. Of the four steels tested in normalized condition it appears that the two alloys containing chromium show greater resistance to weakening by increase in temperature to about $550^{\circ} \mathrm{C}\left(1020^{\circ}\right.$ F) than either the plain carbon or $31 / 2$ per cent nickel steels, and at this highest temperature the chromium-vanadium steel is to be preferred from the standpoint of high tensile strength and limit of proportionality.

2. The carbon and $3^{1 / 2}$ per cent nickel steels behave alike with rise in temperature, and at about $550^{\circ} \mathrm{C}\left(\mathrm{IO}_{2} \mathrm{O}^{\circ} \mathrm{F}\right)$ the addition of the nickel appears to have a very small effect on the tensile properties of the carbon steel.

\footnotetext{
${ }^{\ominus}$ Zay Jeffries, "Effect of temperature, deformation, and grain size on the mechanical properties of metals," Trans. A. I. M. E., 60, D. 474; I919.
} 
3. At $550^{\circ} \mathrm{C}\left(1 \mathrm{O} 20^{\circ} \mathrm{F}\right)$ the strength and limit of proportionality of the chromium-vanadium steel are more than twice that of the carbon steel, while the ductility of the former, as measured by elongation and reduction of area, is about half that of the latter, though still quite high. However, at all temperatures below about $475^{\circ} \mathrm{C}\left(885^{\circ} \mathrm{F}\right)$ the strength of the nickel-chromium steel is greater than that of the chromium-vanadium and both show higher strength values throughout the range 20 to $550^{\circ} \mathrm{C}$ ( 70 to ${ }_{1020^{\circ}} \mathrm{F}$ ) than the carbon or $3 \frac{\mathrm{T}}{2}$ per cent nickel steels.

4. While no general statements regarding types of fractures can be made with certainty, the tendency of carbon and $3 \frac{\mathrm{T}}{2}$ per cent nickel steels at about $550^{\circ} \mathrm{C}\left(1020^{\circ} \mathrm{F}\right)$ is to follow the grain boundaries, while at the same temperature the fractures of the chromium-vanadium steel appear largely transcrystalline.

\section{ACKNOWLEDGMENT}

In conclusion acknowledgment is made to C. E. Lermond, mechanical draftsman, Ordnance Department, and T. E. Hamill, laboratory apprentice, Bureau of Standards, for carrying out the greater part of the routine tensile tests reported in this paper.

Washington, August I, I92I. 